\title{
BMJ Open Epidemiology of venous thromboembolism in people with active tuberculosis: a systematic review and meta-analysis protocol
}

\author{
Philippe Armel Awana, ${ }^{1}$ Celestin Danwang (D) , ${ }^{2,3}$ Joel Noutakdie Tochie (D) , ${ }^{4}$ \\ Jean Joel Bigna (iD ${ }^{5}$
}

To cite: Awana PA, Danwang C, Tochie JN, et al. Epidemiology of venous thromboembolism in people with active tuberculosis: a systematic review and metaanalysis protocol. BMJ Open 2019;9:e031402. doi:10.1136/ bmjopen-2019-031402

- Prepublication history and additional material for this paper are available online. To view these files, please visit the journal online (http://dx.doi. org/10.1136/bmjopen-2019031402).

Received 02 May 2019 Revised 09 September 2019 Accepted 04 October 2019
Check for updates

(c) Author(s) (or their employer(s)) 2019. Re-use permitted under CC BY-NC. No commercial re-use. See rights and permissions. Published by BMJ.

For numbered affiliations see end of article.

Correspondence to Dr Celestin Danwang; danram07@yahoo.fr

\section{ABSTRACT}

Introduction In recent years, a hypothesis has been raised that people with tuberculosis are at risk for developing venous thromboembolism (VTE). However, much remains to be understood about the interplay between VTE and tuberculosis. Therefore, through a global systematic review and meta-analysis, the current study aims at investigating the association between exposure to active tuberculosis and risk of VTE, and determining the prevalence and incidence of VTE in people with active tuberculosis.

Methods and design We will include cohort studies, case-control and cross-sectional studies performed among adults ( $>15$ years) with active tuberculosis. EMBASE, Medline, Global Index Medicus and Web of Knowledge will be searched for relevant records published until 31 August 2019, without any language restriction. The review will be reported according to the Meta-analysis Of Observational Studies in Epidemiology guidelines. After screening of titles and abstracts, study selection, data extraction and risk of bias assessment; we shall assess the studies individually for clinical and statistical heterogeneity. Random-effect meta-analysis will be used to pool studies judged to be clinically homogenous. The Egger's test and visual inspection of funnel plots will be used to assess publication bias. Results will be presented by country and region.

Ethics and dissemination Since primary data are not collected in this study, ethical approval is not required. This review is expected to provide relevant data on the epidemiology of VTE in people with tuberculosis. The final report will be published in a peer-reviewed journal. PROSPERO registration number CRD42019130347.

\section{INTRODUCTION}

Venous thromboembolism (VTE) is the third most common cardiovascular disease worldwide and one of the major contributor to disability-adjusted life years lost. ${ }^{1-3}$ It usually appears in people having another baseline disease and is most of the time therefore known as a comorbidity. ${ }^{3}$ VTE which comprised deep venous thrombosis and pulmonary embolism have a global estimated
Strengths and limitations of this study

- This will be the first systematic review summarising data on occurrence of venous thromboembolism in patients having tuberculosis.

- Rigorous methods and robust statistical analysis will be used to minimise bias and provide accurate data.

- No language restriction will be applied, hence, allowing to include the maximum number of studies in this review.

- Limited number of studies on the topic in low/ middle-income counties may represent an important shortcoming.

incidence rate between 1 and 2 per 1000 persons/year in the general population. ${ }^{1}$

The major risk factors of VTE include surgery, pregnancy and postpartum, cancer, oral contraceptive use and prolonged immobilisation. ${ }^{4}$ Risk factors of VTE are those associated with one of the elements describe by Virshow's triad: hypercoagulability, vessel wall injury and stasis. ${ }^{5}$ Recently, the interplay between infections and thrombosis have been raised. ${ }^{367}$ The mechanism may be explained by inflammation which may unbalance the coagulation pathways therefore leading to thrombosis. ${ }^{3}$

Tuberculosis which is one of the most prevalent infectious diseases worldwide, has been described to be associated with high prevalence of VTE in some recent primary studies. ${ }^{68}$ The pathogenesis involved in the development of VTE in patients with tuberculosis (TB) is unclear. However, the Virchow's triad is thought to be at the core of the pathophysiology. ${ }^{9}$ Although the high burden of TB and VTE is clearly known, and evidence suggest a plausible association between TB and VTE, ${ }^{6} 810-12$ the relationship between them is still not well understood. Accordingly, we propose this global systematic review and meta-analysis protocol to critically 
synthesise current evidence on this association. This study will provide evidence-based and useful data that may raise an awareness in healthcare providers, researchers, policymakers and stakeholders for improved detection, overall proper management and efficient control of VTE in the global population with active TB.

\section{Review question}

What is the epidemiology of VTE in the global population of people with active tuberculosis?

\section{Objectives}

This systematic review and meta-analysis aim at:

1. Determining the incidence and prevalence of VTE in the global population having active TB.

2. Investigating the association between exposure to active TB and risk of VTE.

\section{METHODS AND DESIGN}

This systematic review and meta-analysis will be reported in conformity with the Meta-analysis Of Observational Studies in Epidemiology guideline. ${ }^{13}$ The Preferred Reporting Items for Systematic Review and MetaAnalysis for Protocol (PRISMA-P) was used to report this protocol. ${ }^{14}$ The PRISMA-P checklist is attached as online supplementary file 1 .

\section{Criteria for considering studies for the review}

TB cases will be diagnosed on the basis of WHO criteria. ${ }^{15}$ Pulmonary embolism will be defined as the presence of a thrombus in the pulmonary vessels diagnosed by either CT-pulmonary angiography, MRI, ventilation-perfusion lung scan or autopsy. ${ }^{16}$ Deep venous thrombosis will be defined as having a thrombosis of the deep venous circulation including limb, pelvic vessels, vena cava or cerebral vein diagnosed by either Doppler echography, venography, MRI or CT-scan. Study where a different definition of VTE would have been used will be retrieved as well and a subgroup analysis will be conducted to assess the effect of the definition used on the overall summary effect.

Specific criteria for estimating the prevalence and incidence of VTE in people with active tuberculosis

1. Population: we will include studies with adults $(>15$ years) having active pulmonary or extrapulmonary drug-susceptible or drug-resistant TB.

2. Outcomes: we will consider studies reporting the prevalence or incidence of VTE, or studies having enough data to compute these estimates, that is, number of cases of VTE with sample size or total persons-time of follow-up.

3. Study design: we will consider cross-sectional and cohort studies.

Specific criteria for investigating the association between exposure to tuberculosis and risk for VTE

1. Population: we will consider adults ( $>15$ years) with or without any specific conditions or diseases.

2. Exposure will be defined as having active TB.
3. Comparator will be defined as people with a confirmed absence of active TB. Patients with 'Confirmed absence of TB' are those with TB diagnosis negative using the same method of diagnostic as per those with active TB.

4. Outcome should be the presence of any VTE including pulmonary embolism or deep venous thrombosis.

5. We will consider cross-sectional, case-control and cohort studies. For cohort studies, we will consider studies in which both patients with TB (exposed group) and patients without TB (non-exposed group) will be included; and where proportion of patients with VTE was reported in both groups. For case-control studies, we will consider studies in which both patients with VTE (cases) and patients without VTE (control group) will be included; and where proportion of patients with TB was reported in both groups. For cross-sectional studies, data will be considered as reported.

\section{Search strategy for identifying relevant studies}

The search strategy will be conducted as follows.

\section{Bibliographic database searches}

Relevant records will be identified by searching EMBASE, PubMed, Global Index Medicus and Web of Knowledge from inception to 31 August 2019. Text words and medical subject heading terms related to TB and VTE will be used including: 'venous thromboembolism', 'venous thrombosis', 'pulmonary embolism', 'deep venous thrombosis', 'Tuberculosis', 'TB' and 'Mycobacterium' . The online supplementary file 2 shows the full search strategy for PubMed that will be adapted to fit with other databases. No language restriction will be applied. For articles published in a language other than English and French, an experienced translator in the concerned language will be contacted for translation.

\section{Searching for other sources}

We will scan the references of all relevant articles for additional data sources missed during our search, and their full-texts will be retrieved. References of pertinent reviews will also be scanned.

\section{Selection of studies for inclusion in the review}

All references identified after implementation of the searched strategy will be imported inside the Endnote software. All records obtained from various databases will be combined in a single Endnote library, and the duplicates will be removed. Two reviewers (CD and AA) will independently evaluate the studies obtained from the searches, using an assessment form to ensure that the selection criteria are reliably applied. These reviewers will screen the titles and abstracts of papers obtained, after which the full texts of potentially eligible papers will be retrieved by one reviewer (CD). The two reviewers will independently review the full text of each potentially eligible study, compare their results and resolve any discrepancy by discussion. For duplicates, studies published in more than one report, the one reporting the largest sample size will be considered. Studies with 
inaccessible full text either online or from the corresponding author will be excluded.

\section{Assessment of methodological quality and reporting of data}

Methodological quality and risk of bias of included studies will be assessed using the Risk Of Bias In Nonrandomised Studies-of Interventions tool for studies that will be included for investigating the association between TB and VTE. ${ }^{17}$ For studies that will be included for prevalence/incidence, we will use the risk of bias tool developed by Hoy and colleagues. ${ }^{18}$

\section{Data extraction and management}

A data extraction form will be used to collect information on the surname of the first author, year of publication, country where the study was conducted, study design, study area (rural, urban), sampling method, timing of data collection, mean or median age, proportion of males, specific characteristics of the study population, type of treatment regimen, location of the TB infection (extrapulmonary, pulmonary), sample size, total persontime follow-up, number of cases of VTE, ascertainment of active TB and VTE. For multinational studies, the data will be reported for the individual countries. Where it will be impossible to disaggregate data for such studies by country, the available data will be presented as a single study, and the individual countries which participated in the study will be reported. We will exclude studies in which relevant data are impossible to extract even after contacting the corresponding author.

\section{Data synthesis and analysis}

For measuring the association between exposure to active tuberculosis and risk for VTE, a meta-analysis using the random-effects method of DerSimonian and Laird will be performed to pool weighted ORs of risk estimates. ${ }^{19}$ Counter-enhanced funnel plot (if $\geq 10$ studies) and Harbord test (if $\geq 3$ studies) will be done to assess the presence of publication bias. ${ }^{20}$ ORs will be reported with their 95\% CIs, and $95 \%$ prediction intervals.

For prevalence/incidence synthesis, unadjusted prevalence and incidence with their standard errors for each study will be recalculated based on the information of crude numerators and denominators provided by individual studies. The variance of the study-specific prevalence will be stabilised with the Freeman-Tukey double arc-sine transformation, ${ }^{21}$ before pooling the data using a random-effects meta-analysis model. All pooled estimate will be reported with their $95 \% \mathrm{CI}$ and $95 \%$ prediction interval. Heterogeneity will be assessed using the $\chi^{2}$ test on Cochran's $Q$ statistic, and quantified by calculating $\mathrm{I}^{2} .^{22}$ Values of $25 \%, 50 \%$ and $75 \%$ for $\mathrm{I}^{2}$ will, respectively, represent low, medium and high heterogeneity. We will assess the presence of publication bias using funnel plots inspection (if $\geq 10$ studies) and the Egger's test (if $\geq 3$ studies). ${ }^{23}$ When they will be enough data, metaregression and subgroup analyses will be performed to investigate the possible sources of heterogeneity using the aforementioned variables and the study quality. We plan to do a subgroup analysis according to: population type, location of tuberculosis (pulmonary or extrapulmonary), WHO region, income level of the countries where the study was conducted (high, middle and low-incomes countries) and the definition used to diagnose VTE. In case of substantial clinical heterogeneity, a narrative summary of findings will be done. The inter-rater agreement for study inclusion between investigators will be assessed using Cohen's $\kappa$ coefficient. ${ }^{24}$ Data analyses will be done using the 'meta' package of the statistical software R V.3.5.1.

\section{Presentation and reporting of results}

The study selection process will be summarised using a flow diagram. Quantitative data will be presented in tables of individual studies, and in summary tables, and forest plots where appropriate. The quality scores and risk of bias for each eligible study will be reported accordingly.

\section{Patient and public involvement}

Patients and the public were not involved in the design or planning of the study.

\section{Potential amendments}

We do not plan to modify the protocol to avoid reporting bias. However, if necessary, any amendment in the review process will be reported for transparency.

\section{ETHICS AND DISSEMINATION}

Since primary data are not collected in this study, ethical approval is not required. This review is expected to provide accurate data on the occurrence of VTE in patients having tuberculosis. The final report will be published in a peer-reviewed journal.

\section{Review status}

Preliminary searches.

Author affiliations

${ }^{1}$ Department of Radiology, Jamot Hospital, Yaoundé, Cameroon

${ }^{2}$ Institute of Experimental and Clinical Research, Université Catholique de Louvain, Bruxelles, Belgium

${ }^{3}$ Department of Surgery and Specialities, Faculty of Medicine and Biomedical Sciences, University of Yaoundé I, Yaoundé, Cameroon

${ }^{4}$ Department of Anesthesiology and Critical Care Medicine, Faculty of Medicine and Biomedical Sciences, University of Yaoundé I, Yaoundé, Cameroon

${ }^{5}$ Department of Epidemiology and Public Health, Centre Pasteur of Cameroon, Yaoundé, Cameroon

Contributors PAA had the idea. CD and PAA designed and conceived the protocol. CD drafted the manuscript. PAA, JNT and JJB critically revised the manuscript for methodology and intellectual content. CD and JJB are the guarantors of the review. All authors approved the final version of this manuscript.

Funding The authors have not declared a specific grant for this research from any funding agency in the public, commercial or not-for-profit sectors.

Competing interests None declared.

Patient consent for publication Not required. 
Provenance and peer review Not commissioned; externally peer reviewed.

Open access This is an open access article distributed in accordance with the Creative Commons Attribution Non Commercial (CC BY-NC 4.0) license, which permits others to distribute, remix, adapt, build upon this work non-commercially, and license their derivative works on different terms, provided the original work is properly cited, appropriate credit is given, any changes made indicated, and the use is non-commercial. See: http://creativecommons.org/licenses/by-nc/4.0/.

\section{ORCID iDs}

Celestin Danwang http://orcid.org/0000-0002-7976-4331

Joel Noutakdie Tochie http://orcid.org/0000-0002-8338-2467

Jean Joel Bigna http://orcid.org/0000-0001-8018-6279

\section{REFERENCES}

1 Scheres LJJ, Lijfering WM, Cannegieter SC. Current and future burden of venous thrombosis: not simply predictable. Res Pract Thromb Haemost 2018;2:199-208.

2 Danwang C, Temgoua MN, Agbor VN, et al. Epidemiology of venous thromboembolism in Africa: a systematic review. J Thromb Haemost 2017;15:1770-81.

3 Epaulard O, Foote A, Bosson J-L. Chronic infection and venous thromboembolic disease. Semin Thromb Hemost 2015;41:644-9.

4 Heit JA. Epidemiology of venous thromboembolism. Nat Rev Cardiol 2015;12:464-74.

5 Bennett PC, Silverman SH, Gill PS, et al. Peripheral arterial disease and Virchow's triad. Thromb Haemost 2009;101:1032-40.

6 Dentan C, Epaulard O, Seynaeve D, et al. Active tuberculosis and venous thromboembolism: association according to international classification of diseases, ninth revision hospital discharge diagnosis codes. Clin Infect Dis 2014;58:495-501.

7 Chaudhary A, Desai U, Joshi JM. Venous thromboembolism due to hyperhomocysteinaemia and tuberculosis. Natl Med J India 2017;30:139-41.

8 Borjas-Howard JF, Bierman WFW, Meijer K, et al. Venous thrombotic events in patients admitted to a tuberculosis centre. QJM Mon J Assoc Physicians 2017;110:215-8.
9 Gupta A, Dixit R. Pulmonary tuberculosis: a neglected risk factor for deep venous thrombosis. Int J Mycobacteriol 2017;6:184-6.

10 Gupta KB. Thromboembolism in tuberculosis: a neglected comorbidity. Indian J Chest Dis Allied Sci 2014:56:5-6.

11 Gupta A, Mrigpuri P, Faye A, et al. Pulmonary tuberculosis - An emerging risk factor for venous thromboembolism: A case series and review of literature. Lung India 2017;34:65-9.

12 Ogeng'o JA, Obimbo MM, Olabu BO, et al. Pulmonary thromboembolism in an East African tertiary referral hospital. $J$ Thromb Thrombolysis 2011;32:386-91.

13 Stroup DF, Berlin JA, Morton SC, et al. Meta-Analysis of observational studies in epidemiology: a proposal for reporting. meta-analysis of observational studies in epidemiology (moose) group. JAMA 2000;283:2008-12.

14 Moher D, Shamseer L, Clarke M, et al. Preferred reporting items for systematic review and meta-analysis protocols (PRISMA-P) 2015 statement. Syst Rev 2015;4:1.

15 WHO. Global tuberculosis report 2017, 2017. Available: http://www. who.int/tb/ publications/global_report/en/ [Accessed 27 Mar 2019].

16 Di Nisio M, Lee AYY, Carrier M, et al. Diagnosis and treatment of incidental venous thromboembolism in cancer patients: guidance from the SSC of the ISTH. J Thromb Haemost 2015;13:880-3.

17 Risk of Bias Tools. ROBINS-I tool(Risk of bias in non-randomized studies - of interventions). Available: https://sites.google.com/site/ riskofbiastool/welcome/home [Accessed 12 Mar 2019].

18 Hoy D, Brooks P, Woolf A, et al. Assessing risk of bias in prevalence studies: modification of an existing tool and evidence of interrater agreement. J Clin Epidemiol 2012;65:934-9.

19 DerSimonian R, Laird N. Meta-analysis in clinical trials revisited. Contemp Clin Trials 2015;45:139-45.

20 Harbord RM, Egger M, Sterne JAC. A modified test for small-study effects in meta-analyses of controlled trials with binary endpoints. Stat Med 2006;25:3443-57.

21 Miller JJ. The inverse of the Freeman - Tukey double Arcsine transformation. Am Stat 1978;32:4

22 Huedo-Medina TB, Sánchez-Meca J, Marín-Martínez F, et al. Assessing heterogeneity in meta-analysis: $Q$ statistic or 12 index? Psychol Methods 2006;11:193-206.

23 Egger M, Davey Smith G, Schneider M, et al. Bias in meta-analysis detected by a simple, graphical test. BMJ 1997;315:629-34.

24 McHugh ML. Interrater reliability: the kappa statistic. Biochem Med (Zagreb) 2012;22:276-82. 